\title{
Voltage Coordination via Communication in Large-Scale Multi-Area Power Systems. Part I: Principal
}

\author{
Mohammad Moradzadeh", a , René Boel ${ }^{2, b}$ \\ ${ }^{1,2}$ Department of Electrical Energy, Systems and Automation, University of \\ Ghent, Technologiepark-Zwijnaarde 914, B-9052 Ghent, Belgium \\ amohammad.moradzadeh@ugent.be, ${ }^{\mathrm{b}}$ rene.boel@ugent.be
}

Keywords: Coordination, optimization, abstraction, voltage control, large-scale multi-area power system

\begin{abstract}
This two-part paper deals with the coordination of the control actions in a network of many interacting components, where each component is controlled by independent control agents. As a case study we consider voltage control in large electric power systems, where ever-increasing pressures from the liberalization and globalization of the electricity market has led to partitioning the power system into multiple areas each operated by an independent Transmission System Operator (TSO). Coordination of local control actions taken by those TSOs is a very challenging problem as poorly coordinated operation of TSOs may endanger the power system security by increasing the risk of blackouts. This coordination problem involves many other issues such as communication, abstraction and last but not least optimization. This first part of the paper is devoted to the principals of the coordination control, addressing some of those issues using as a case study the problem of coordination control for avoiding voltage collapse in large-scale multi-area power systems.
\end{abstract}

\section{Introduction}

The traditional power systems undergo major changes due to recent trends in the power industry, especially deregulation of electrical power systems and the growing concern of ever-increasing internationalization and liberalization in the competitive electricity market. This results in multiarea systems operated by several TSOs (Transmission System Operators) [1-2]. These TSOs are typically interconnected through power transmission corridors which carry heavy power flows. The power flow in the tie-lines of interconnected power systems may be controlled to ensure economical benefits/constraints as well as to avoid possible voltage collapses [3]. The aggregated utilities economically establish a common open electricity market and technically ensure a greater security margin, through sharing of active/reactive power reserves. But voltage control, due to its physical characteristic, still remains a prerogative of the local utilities. The voltage and current values at boundary buses, and hence the flow of active and reactive power over the tie-lines depend on the control actions taken by different involved parties. TSOs in practice only have local knowledge about their own model and may only know about active/reactive power flow or the voltage profile at interconnections. Thus, secure operation of multi-area power systems requires appropriate coordination of control actions taken by (at least adjacent) TSOs. In the case of simple and small interconnected power system, e.g. power systems consisting of at most two areas, or in the conventional radial power systems with the integration of distributed generation resources (DERs), the coordination may be obtained by heuristic ad-hoc schemes based on off-line assessment of utilities [4]. As the size of the network increases, or in the case of complex meshed structures, coordination can be a complicated challenge. Local control actions in one area may have strong 
influence on the system variables of its neighboring utilities initiating other, possibly undesirable, control actions by these neighbors.

Note that frequency control is linked to generation-load imbalance, frequency being a global variable, while voltage control is linked to electrical distance between generation and load, voltage being a local variable. Therefore, voltage control is a logical application for coordination control.

As far as we know, little attention has been paid on devising a truly model-based coordinating feedback voltage control of complex interconnected power systems. This coordination is a major challenge involving many issues such as what information to communicate, how to avoid the need that each area knows the global model, and the choice of abstraction level of the models. To design a well-performing system-wide coordination control in large-scale multi-area power system, those important issues need to be carefully addressed.

Coordination, achieved typically via exchanging messages on scheduled control actions among TSOs, should be implemented in such a way that each area has its own voltage regulator. This regional coordinator determines the favorable combinations of control actions with respect to the voltage profile of its own area i.e. to maintain the voltage profile at the scheduled level while maintaining the net tie-line interchange from the given area at acceptable values.

The communication and information exchange must also be limited as electricity utilities tend to preserve some prerogative of their own system operation and it may not be acceptable to reliably communicate all the necessary information about operational conditions, scheduled active/reactive power generation pattern, load demand, control actions, objective functions and constraints. Another challenge for TSOs is to approximate the external neighboring utilities model from their (possibly) complex-structured system. Standard abstraction algorithms e.g. Thevenin theorem calculate exact equivalence, while advanced predictive model-based control theory needs an approximated model capable of reflecting the changes occurring in the neighboring areas as a reaction to the neighbor's control actions (communicated) and as a reaction to their own power exchange with neighbors, but without requiring too much computation and without being too sensitive to inaccurately known parameters of the approximated model of the neighbors.

This first part of the paper is organized as follows. Section II, suing a 12-bus meshed power system, illustrates the necessity of coordination for voltage control and shows how local control actions in one area may initiate other, possibly undesirable, control actions by the neighboring areas. Section III compares the main trends in development of coordination schemes in electrical power networks. The basic characteristics and also fundamental limitations of each scheme will be briefly presented. In section IV, the use of communication is explained. The problem of obtaining abstraction for component models will be discussed in section V. Finally, conclusions are provided in section VI.

\section{Example Illustrating the Necessity of Coordination}

This example illustrates why coordination is necessary for voltage control in power systems and how different local control actions can trigger each other. The 12-bus meshed test system, shown in Fig. 1, is taken from [5]. LTCs 1,2 and 3 respectively try to locally maintain the voltage of buses A,B and C within an upper and lower bound e.g. [0.98, 1.02] p.u. Two pairs of "bus voltage-LTC moves" i.e. (LTC 1, Bus A) and (LTC 2, Bus B) are shown in Fig. 2, following the outage of two parallel lines in the location $\mathrm{F}$ at $\mathrm{t}=20 \mathrm{~s}$. Following the fault, both LTCs, after a delay of $10 \mathrm{~s}$, try to restore the corresponding local voltages. As one can see, the voltage of Bus A is already within its safety limit around $t=40 \mathrm{~s}$, but still one extra move around $t=82 \mathrm{~s}$ is observed. This extra move is indeed attributed to the interaction of LTC 2.

The coordination schemes aim to avoid these kinds of interactions among local controllers.

\section{Main Trends in Development of Coordination Schemes}

Roughly speaking, coordination strategies can be classified into three different trends. 


\section{A. Centralized Coordination Schemes}

A centralized coordination unit at the top level of the hierarchy receives information from all TSOs via communication links. All control actions to be taken by TSOs are computed in one single multiparty (possibly multi-objective) optimization problem subject to individual constraints of each single party. The main drawback of centralized coordination in the large-scale multi-area power systems is the huge computational cost, lack of robustness due to requiring global knowledge of the complete model of the overall system, and reliability problems due to possible communication failures. In addition, the decision made by the central coordination unit should be sufficiently "fair" to be accepted by each party. The concept of fairness in the sense of economics has been briefly introduced in [6] where fairness criteria namely "free from envy", "efficiency", "accountability" and "altruism" have been checked for a centralized optimization method of multi-TSO power system. Successful centralized coordination has been reported only for rather small-scale power systems [79].

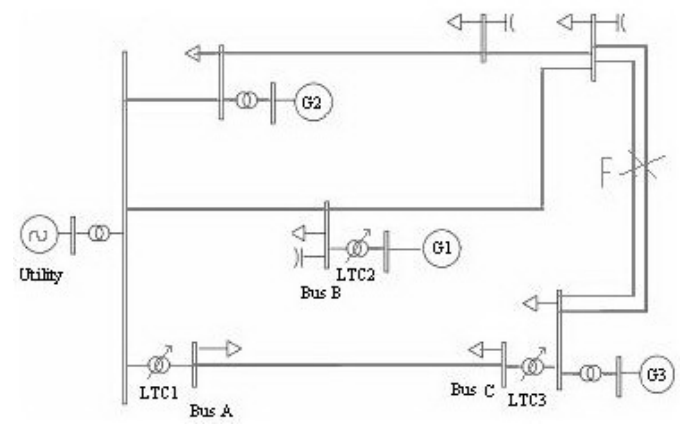

Fig. 1: On-line diagram of a 12-bus power system
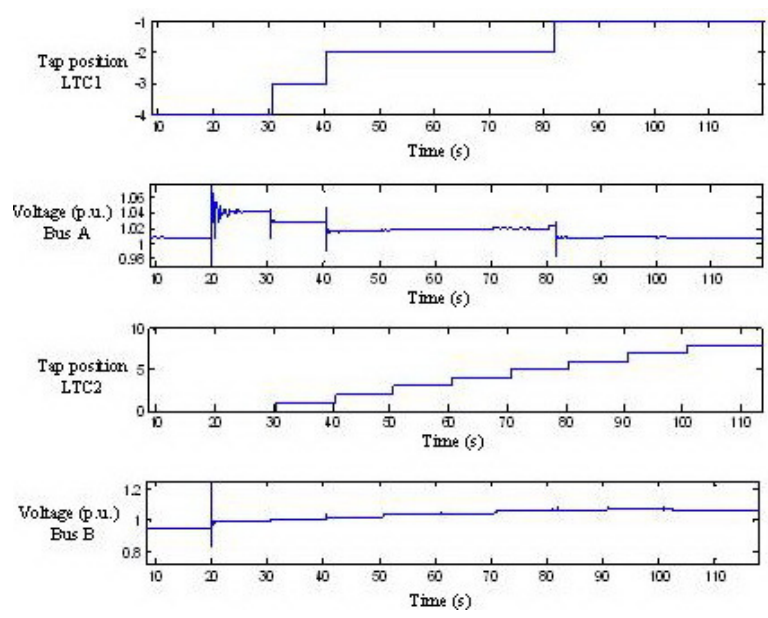

Fig. 2: Bus voltages and the corresponding LTC actions

\section{B. Decentralized schemes (no coordination)}

On the other hand, (purely) decentralized schemes with no information exchange have been proposed to overcome the above-mentioned drawbacks of centralized approaches. TSOs are assumed to be non-overleaping. Each TSO solves its own optimization problem without taking the objective and constraint of neighboring areas into account, and ignoring the interactions among TSOs [10-11]. In the context of large-scale multi-area power systems where the dynamics of the different areas are highly coupled, the decentralized approach may not lead to well-performing system-wide coordination control. The decentrally computed solutions will in general not converge to the (nearly) optimal global centralized performance. It may not even be stable in cases where a stabilizing centralized controller exists. The decentralized optimal solution assigns the least possible cost to every party so that there exist no other solution that reduces at least one cost without 
increasing any other costs (and no constraints are violated). In game theory this is called the Pareto optimal solution.

\section{Distributed coordination schemes}

A recently developed state-of-the-art approach [12-13], a so called distributed coordination scheme relies on partitioning of the large-scale power system into several areas, each area being controlled by an independent TSO. Fig. 3, as an example, shows a physically interconnected 4- area power system namely Nordic32, consisting of Norway, Sweden, Denmark and Finland. Nordic32 is a complex meshed 71-bus 20-machine transmission system. All countries are nowadays deregulated in slightly different ways, and the conventional centralized power dispatching is replaced with the market-based power exchange NordPool [14]. The latter dispatches the power generation among areas, on an hourly basis, according to competitive market rules. Some operational information is shared, from time to time, by the market participants in the NordPool's information system (called Urgent Market Message (UMM)).

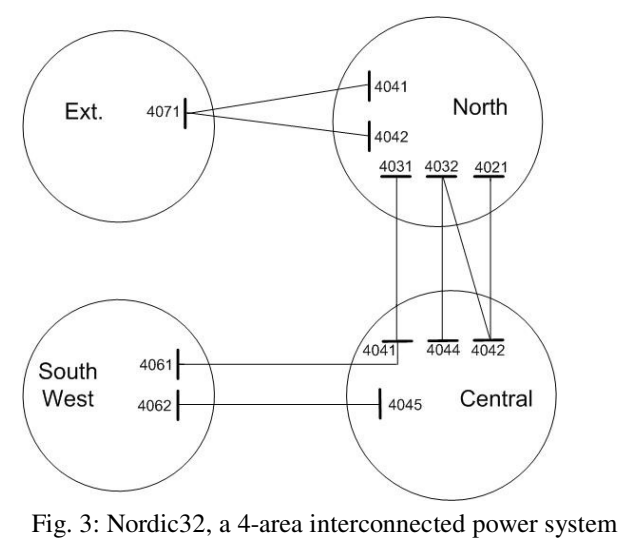

Each TSO is assumed to have exact and detailed information on its own authority (dynamic model and all the local on-line measurements) including voltages and power flows at interconnections; the evolution in the neighboring areas is taken into account only by representing them with an approximately equivalent model. Obviously the parameters of this equivalent model must be updated on-line by appropriate parameter identification techniques as the system's dynamics evolves in time. Furthermore, each TSO is assumed to agree on transmitting some of its on-line information to the neighboring TSOs. Intuitively, the minimal information necessary for achieving acceptable performance includes the local control decisions (solutions of the local optimization problem). In this way, distributed coordination strategies combine the advantages of both centralized and decentralized approaches. TSOs may further negotiate on what additional information exchange should be exchanged. The performance of the distributed coordination schemes may be influenced by two key mechanisms of a) class of approximately equivalent models and b) parameter fitting for the approximated model. Every TSO is assumed to adopt the same type of equivalent models, used to represent its neighboring areas as well as identical parameter identification algorithm to estimate the parameters of the equivalent [15]. Special cases where one (or several) TSOs fail to find a solution for the local optimization problem, due to inevitable violation of the constraint for the set of all available control actions, must be handled by sending request for additional reactive power to supervisor.

The second part of this paper initially applies the Distributed Model Predictive Control (D-MPC) paradigm to the voltage control problem of a 12-bus 3-area power system, assuming that each area knows the entire system model. Simulation results show provided that each TSO is agreed on transmitting some of its online information to the neighboring TSOs, D-MPC can prevent voltage collapse under circumstances where classical uncoordinated controllers fail. However it is equally 
true that the local controllers can not know the complete model of the entire system in detail. For this reason, using the well-known Nordic32 test system, we are currently investigating the D-MPC coordination scheme when each local agent only knows an approximate model of distant neighbring areas. Simulation results and the performance of the D-MPC controller for Nordic32 will be reported in another paper under preparation. Fig. 4 shows a general block-diagram representation of a distributed MPC-based coordination scheme applied to the voltage coordination problem of Nordic32.

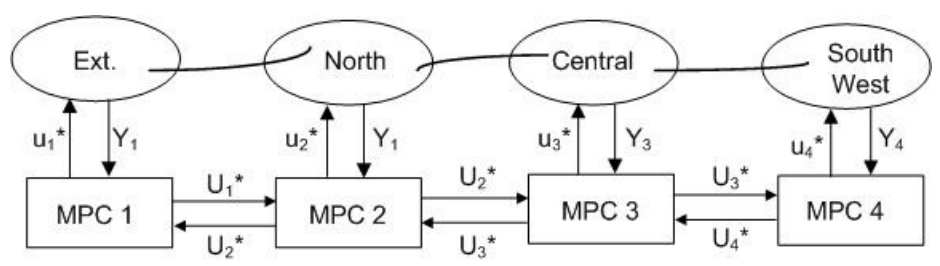

Fig. 4: D-MPC applied to Nordic32

The Central area is the main load center containing 13 LTCs. Each local MPC controls one (or a few) LTCs. This Central area is further decomposed in order to apply D-MPC to coordinate local MPC actions in the Central area.

\section{Communication}

The availability of on-line synchronized phasor measurement units (PMUs) as well as reliable highspeed communication networks enables Wide-Area Monitoring and Control (WAMC) systems [1617] which complement already existing classical SCADA/EMS Supervisory Control And Data Acquisition/Energy Management System) platforms. Traditional SCADA/EMS systems are based on steady-state power flow analysis and have the industry standard resolution of 2-4 seconds. Therefore, this system cannot observe the faster dynamic variables of the power system. The newly developed WAMC/PMU platform ease the dynamic monitoring and control of the TSOs by providing real-time "snapshots" of the power system state variables up to every m-seconds. A sampling interval of $20 \mathrm{~ms}$ is fast enough to evaluate frequency, voltage and current phasors (magnitude and angle) and their correlation with time. The time scale of the long-term voltage control of interest for this paper is typically in the period of several minutes after a disturbance. To emphasize this time resolution difference between PMU and SCADA/EMS technologies, these are often referred to as "MRI" resp. "X-ray" of the power systems.

\section{Abstraction}

Nowadays electrical power systems are typically very complex large-scale geographically widespread networks of components with different TSOs interconnected to each other. A full detailed model of such a system for on-line control, due to its complexity, seems to be economically inefficient and computationally too time-consuming. Furthermore, this becomes practically impossible, even with availability of powerful computational resources, in the context of multi-area power systems where TSOs do not have the full detailed dynamic model of the entire system, but only know the local model of their region of authority. Therefore, network equivalencing procedures, also called network reduction techniques, to properly represent static (steady-state) and dynamic characteristics of the power system models are of great importance. Obviously, the intuitive hypothesis behind the idea of a valid model reduction is that the equivalent (external/reduced) system model should reproduce similar effect as the detailed model on the study (internal/retained) system following all disturbances that can occur in practice.

Corresponding to the steady-state resp. transient performance of the power system following a disturbance, two different types of equivalents namely static resp. dynamic equivalents can be distinguished. 
The classical static external network equivalents are Ward equivalents [18] and REI (Radial, Equivalent and Independent) equivalents [19]. They do not reflect the probable changes/contingencies occurring inside the external network and thus the simulation of the new conditions requires the development of a new static equivalent model. The parameters of these equivalents are normally updated by using a measurement-based estimation (i.e. a recorded history of past observations at interconnections). Therefore, the changes in the external system can be taken into account only after new measurements become available. As static equivalents are generally obtained for a base/reference operating condition, the computed internal states for the other operating conditions (resulting from e.g. changes in the loading level, outage of some components, or control actions) will be inherently erroneous. This is especially important when the external area contains a considerable number of active DG units whose power generation pattern (switching status) varies regularly [20].

On the other hand, classical dynamic equivalent models update their parameters for representing real-time topology changes of the external system. In real-life on-line applications e.g. dynamic security assessment, stability analysis, fault detection, adaptive protection schemes and control design etc. a simple and fast dynamic equivalent is necessary. The parameters of such an equivalent model should be determined using measurements data taken at interconnections of two sub-systems. Roughly speaking, the construction of the dynamic equivalent can be accomplished using reductionbased and identification-based approaches [21].

\section{A. Reduction-Based Approaches}

In reduction-based approaches, the equivalent is typically determined after aggregation of generator terminal buses and the elimination of load buses. Modal techniques [22-23] and coherency analysis [24-26], as the two primary and mostly contemplated techniques, belong to this category. Since these approaches are based on an exact model of components, they are considerably accurate and reliable. Some synchronous generators are observed to tend to swing together after a disturbance. Coherency measures provide a grouping criterion to identify a group of coherent generators, which swing together, and have identical terminal voltage. However, identification of coherent groups and thus aggregation procedure, require a complete set of parameters of the individual components in the external system to estimate the parameters of equivalent aggregated component. This may be problematic in the framework of large-scale multi-area power systems where each TSO preferably should directly derive the equivalent model for its neighboring areas from a set of real-time measurements taken solely at boundary buses of interconnections. In this way, the equivalent model will be independent of the external network size and complexity, and the correct on-line information of the external areas will not be required. Furthermore, coherency criterion seems to fail when applying to the power systems with high penetration of converter-based DG units e.g. fuel cells and photovoltaic where their characteristic is not even determined by electromechanical behavior [20].

\section{B. Identification-Based Approaches}

On the other hand, identification-based approaches determine the dynamic equivalent models by perturbing the internal system by natural/intentional disturbances, and monitoring the response of the assumed approximated system model variables by taking measurements at boundary buses. These real-time measurements will be compared with those computed corresponding signals with the approximated system model applied. The equivalent model will then be adjusted so as to match those two signals (real-time measurement and corresponding computed value) to each other as well as possible. Identification-based approaches are more desirable in the context of multi-TSO systems with the limited access to only boundary information. However, since these approaches yield an approximated model determined by noisy measurements taken only at boundary buses, rather than exact physical model given by reduction-based approach, they seem to be less accurate. Furthermore, advanced control theories e.g. model predictive control etc. call for dynamic modelbased equivalents whose parameters, to be determined with least required measurements data taken 
at interconnections, can reflect the changes in the external network (over the finite control/prediction horizons ahead) [27].

\section{Summary}

The main trends in development of coordination schemes in large-scale multi-area electrical power systems particularly for voltage control problem are presented in this first part of the paper. The issues of communication, abstraction and optimization are highly linked to coordination control. These issues have been briefly addressed in the paper. By way of summery, the following conclusions can be drawn and must be taken into account when applying distributed model-based coordination schemes to the coordinated voltage control problem in large-scale multi-area power systems.

- Distributed cooperative-based coordination schemes fit best the requirements of multi-area large-scale power systems where each TSO optimizes its own utility in a greedy way without taking the cost and constraints of the neighbors into account.

- The inherent feedback structure of the D-MPC provides enough robustness against modeling errors e.g. uncertainties in the load modeling as well as measurement inaccuracies.

- The minimum exchange of information among TSOs necessary for achieving acceptable coordination includes the local control decisions.

- Time-variant nature of the power system calls for dynamic equivalents whose parameters can be estimated via identification using measurements taken solely at boundary buses of interconnections.

- In order to obtain an equivalent model for neighboring areas in the multi-area power systems, identification-based approaches is more desirable than reduction-based approaches since it may be utilized with limited amount of information at boundary buses.

\section{References}

[1] Union for the Coordination of Transmission of Electricity (UCTE), "UCTE Operation Handbook," 2009.

[2] B. Fardanesh, "Future trends in power system control," IEEE Computer Applications in Power, vol. 15 , no. 3, pp. 24-31, July 2002.

[3] A.M. Azmy, "Optimal Power Flow to Manage Voltage Profiles in Interconnected Networks Using Expert Systems,” IEEE Trans. Power Systems, vol.22, no.4, pp.1622-1628, Nov. 2007.

[4] T. V. Cutsem, C. Vournas, "Voltage Stability of Electric Power Systems", Springer, 1998.

[5] J. Shan, U.D. Annakkage and A.M. Gole , "A platform for validation of FACTS models," IEEE Trans. on Power Delivery, vol.21, no.1, pp. 484- 491, Jan. 2006.

[6] Y. Phulpin, M. Begovic, M. Petit and et al., "A fair method for centralized optimization of multi-TSO power systems," International Journal of Electrical Power \& Energy Systems, vol. 31, issue 9, pp. 482-488, Oct.2009.

[7] H. Seyedi and M. Sanaye-Pasand, "New centralised adaptive load-shedding algorithms to mitigate power system blackouts," in IEE Proc. Generation, Transmission \& Distribution, vol.3, no.1, pp.99-114, Jan. 2009.

[8] N. Nimpitiwan and C. Chaiyabut, "Centralized Control of System Voltage/Reactive Power Using Genetic Algorithm," in Proc. International Conference on Intelligent Systems Applications to Power Systems, pp.1-6, 5-8 Nov. 2007.

[9] A.G. Beccuti, T.H. Demiray, G. Andersson and et al. "A Lagrangian Decomposition Algorithm for Optimal Emergency Voltage Control," IEEE Trans. Power Syst., vol.25, no.4, pp.17691779, Nov. 2010. 
[10] B. Tyagi and S.C. Srivastava, "A decentralized automatic generation control scheme for competitive electricity markets," IEEE Trans. Power Syst., vol.21, no.1, pp. 312- 320, Feb. 2006.

[11] K. Tanaka, M. Oshiro, S. Toma and et. al "Decentralised control of voltage in distribution systems by distributed generators," in IEE Proc. Generation, Transmission \& Distribution, vol.4, no.11, pp.1251-1260, Nov. 2010.

[12] E. Camponogara, D. Jia, B.H. Krogh and et al., "Distributed model predictive control," Control Systems Magazine, IEEE, vol.22, no.1, pp.44-52, Feb. 2002.

[13] A.N. Venkat, I.A. Hiskens, J.B. Rawlings and et al. "Distributed MPC Strategies With Application to Power System Automatic Generation Control,", IEEE Trans. Control Systems Technology, vol.16, no.6, pp.1192-1206, Nov. 2008.

[14] S. Karkkainen and E. Lakervi, "Liberalisation of electricity market in Finland as a part of Nordic market," in IEE Proc. Generation, Transmission and Distribution, vol.148, no.2, pp.194-199, Mar 2001.

[15] Y. Phulpin, M. Begovic, M. Petit and et al. "Evaluation of Network Equivalents for Voltage Optimization in Multi-Area Power Systems," IEEE Trans. Power Syst., vol.24, no.2, pp.729743, May 2009.

[16] M. Zima, M. Larsson, P. Korba and et al. "Design Aspects for Wide-Area Monitoring and Control Systems," in Proc. of the IEEE, vol.93, no.5, pp.980-996, May 2005.

[17] J. De La Ree, V. Centeno, J.S. Thorp and et al. "Synchronized Phasor Measurement Applications in Power Systems," IEEE Trans. Smart Grid, vol.1, no.1, pp.20-27, June 2010.

[18] J. B. Ward, "Equivalent Circuits for Power-Flow Studies," Trans. of the American Institute of Electrical Engineers, vol.68, no.1, pp.373-382, July 1949.

[19] P. Dimo, "Nodal Analysis of Power Systems", Abacus Press, Kent, England, 1975.

[20] A.M. Azmy, I. Erlich, "Identification of dynamic equivalents for distribution power networks using recurrent ANNs," in Proc. Power Systems Conference and Exposition, vol.1, pp. 348- 353, 10-13 Oct. 2004.

[21] T. Singhavilai, O. Anaya-Lara, K.L. Lo, "Identification of the dynamic equivalent of a power system," in Proc. 44th International Universities Power Engineering Conference (UPEC), pp.1-5, 1-4 Sept. 2009.

[22] J.M. Undrill, A.E. Turner, "Construction of Power System electromechanical Equivalents by Modal Analysis," IEEE Trans. Power Apparatus and Systems, vol.PAS-90, no.5, pp.2049-2059, Sept. 1971.

[23] J. M. R. Arredondo, R. G. Valle, "An optimal power system model order reduction technique," International Journal of Electrical Power \& Energy Systems, vol. 26, issue 7, pp. 493-500, Sep. 2004.

[24] R. Podmore, "Identification of Coherent Generators for Dynamic Equivalents,", IEEE Trans. Power Apparatus and Systems, vol.PAS-97, no.4, pp.1344-1354, July 1978.

[25] F. Wu, N. Narasimhamurthi, "Coherency identification for power system dynamic equivalents," IEEE Trans. Circuits and Systems, vol.30, no.3, pp. 140- 147, Mar 1983.

[26] J. Sung-Kwan, L. Chen-Ching, L.E. Jones and et al. "Coherency and aggregation techniques incorporating rotor and voltage dynamics," IEEE Trans. Power Syst., vol.19, no.2, pp. 1068- 1075, May 2004.

[27] A.M. Stankovic, A.T. Saric, M. Milosevic, "Identification of nonparametric dynamic power system equivalents with artificial neural networks," IEEE Trans. Power Syst., vol.18, no.4, pp. 1478- 1486, Nov. 2003. 